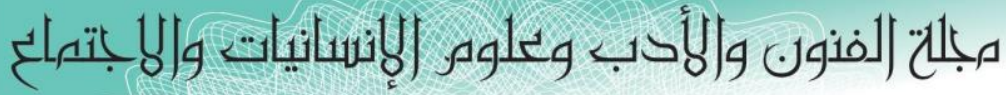

Journal of Arts, Literature, Humanities and Social Sciences

ISSN online: 2414 - 3383

ISSN print: 2616 - 3810

العدد (39) حزيران - يونيو 2019

\title{
Comparison between two Estimators by Using Process Capability with Application
}

\author{
Dler Hussein Kadir \\ Department of Business Administration, Cihan University-Erbil, Kurdistan Region, Iraq \\ Department of Statistics, Salahaddin University-Erbil, Kurdistan Region, Iraq
}

\section{Dlshad Mahmood Saleh}

Department of Business Administration,Paitaxt Private Technical Institute ,Erbil, Kurdistan Region, Iraq Department of Statistics, Salahaddin University-Erbil, Kurdistan Region, Iraq

\section{Dashty Ismil Jamil}

Department of Health Administration, Lebanese French University -Erbil, Kurdistan Region, Iraq

\begin{abstract}
Process capability is the long-term performance level of the process after it has been brought under statistical control. In other words, process capability is the range over which the natural variation of the process occurs as determined by the system of common causes.

Robust estimator is an estimator which is insensitive to changes in the underlying distribution and also resistant against the presence of outliers.

In this paper proposes the using Downton estimator in the process capability indices and compare with $(\hat{\sigma})$ estimator in the Process Capability Indices to justify the efficiency. Concluded that in this study, Downton estimator have better property than the other $(\hat{\sigma})$ estimator because that the process Capability Indices values based on Downton estimator greater than the process Capability Indices values based on $(\hat{\sigma})$ estimator.We also found the process Capability Indices based on Downton estimator can be used as an alternative instead of the process Capability Indices values based on $(\hat{\sigma})$ estimator.
\end{abstract}




\section{INTRODUCTION}

Process capability measurements allow us to summarize process $\mathrm{c}$

apability in terms of meaningful percentages and metrics to predict the extent to which the process will be able to hold tolerance or customer requirements.

You can compute how often the process will meet the specification or the expectation of your customer, you may learn that bringing your process under statistical control requires fundamental changes - even redesigning and implementing a new process that eliminates the sources of variability now at work.

It helps you choose from among competing processes, the most appropriate one for meeting customers' expectation knowing the capability of your processes. You can specify better the quality performance requirements.

The classical process capability ratio (Kane, 1986):

$$
C_{p}=\frac{U S L-L S L}{6 \hat{\sigma}}
$$

Where

$\hat{\sigma}=\frac{\bar{s}}{c_{4}}$

Factors for Computing Central Lines and ControlLimits based on sample size (n) .

The $C_{p k}$ index proposed by (Sullivan, 1985) is a measure of the capability of a process in relation to the process average. It is based on the distance between the process average and the closest specification limit, and is defined as:

$$
\begin{aligned}
& C_{p k}=\min \left(\frac{U S L-\overline{\bar{x}}}{3 \hat{\sigma}}, \frac{\overline{\bar{x}}-L S L}{3 \hat{\sigma}}\right) \ldots . \\
& C_{p k_{U}}=\frac{U S L-\overline{\bar{x}}}{3 \hat{\sigma}} \\
& C_{p k_{L}}=\frac{\overline{\bar{x}}-L S L}{3 \hat{\sigma}}
\end{aligned}
$$

(Chan, 1988) proposed another index, called $C_{p m}$ which is defined as: 
$C_{p m}=\frac{U S L-L S L}{6 \sqrt{\hat{\sigma}^{2}+(\bar{x}-T)^{2}}}$

(Kotz, S. \& Johnson.; 1992) proposed another index, called, $C_{p m k}$ which is defined as:

$C_{p m k}=\frac{\min (U S L-\bar{x}, \bar{x}-L S L)}{3 \sqrt{\hat{\sigma}^{2}+(\bar{x}-T)^{2}}}$

\section{Robust Scale Downton Estimator.}

The Robust Scale Downton estimator was first introduced by Downton as an estimator for the standard deviation of a normal population. (Barnett, Mullen \& Saw) showed that Downton statistic is an unbiased estimator of $(\sigma)$. Let $X_{1}, X_{2}, \ldots \ldots$, $X n$ represent a random sample of size $\mathrm{n}$ from a normal distribution with mean $\mu$ and standard deviation $(\sigma)$; that is, let $X \sim N(\mu, \sigma 2)$ and the corresponding order statistic be denoted by $X_{(1)}, X_{(2)}, \ldots \ldots . X(n)$ where $X(1) \leq X(2) \ldots \ldots . \leq X(n)$. The Downton estimator is defined (Downton, F. 1966) (Abbasi, S.A. \& Miller, A. 2013) as:

$$
\begin{aligned}
& D=\sqrt{\pi} \sum_{i=1}^{n} \frac{(2 i-n-1) x_{i}}{n(n-1)} \\
& D=\frac{2 \sqrt{\pi}}{n(n-1)} \sum_{i=1}^{n}\left[\left(i-\frac{1}{2}(n+1)\right] x_{i}\right]
\end{aligned}
$$

Where the unbiased estimator for $(\sigma)$ is given as $\hat{\sigma}=\bar{D}$, which is used in this study.

$$
\bar{D}=\frac{\sum_{i=1}^{m} D_{i}}{m}
$$

Where

$\mathrm{m}$ : is a preliminary number of the subgroups.

$\mathrm{D}$ : is defined as in (5). 


\section{Process Capability Indices Based on Robust Scale Downton Estimator}

Let $x_{i j}$ represent a random sample of size $\mathrm{n}$ taken over $\mathrm{m}$ subgroup, $\mathrm{i}=1,2 \ldots$, $\mathrm{n}$ and $\mathrm{j}=1,2, \ldots, \mathrm{m}$. The sample are assumed to be independent and taken from a continuous identical distribution functions. If $\left(\sigma^{2}\right)$ is unknown, then an unbiased estimate of $\left(\sigma^{2}\right)$ is the sample variance $\left(s^{2}\right)$. In practice, the normality assumption is often violated by real life data, therefore, using $\left(s^{2}\right)$ as an estimate of $\left(\sigma^{2}\right)$ will affect the process capability indices and thus this might leads to wrong signal and invalid inference. The $C_{p}$ index based on Downton estimator will be defined by (Adeoti, O. A, Olaomi, J.O. \& Adekeye, K.S. 2016):

$$
C_{p}=\frac{U S L-L S L}{6(\bar{D})}
$$

The $C_{p k}$ index based on Downton estimator will be defined by

$$
C_{p k}=\min \left(\frac{U S L-\overline{\bar{x}}}{3 \bar{D}}, \frac{\overline{\bar{x}}-L S L}{3 \bar{D}}\right)
$$

The $C_{p m}$ index based on Downton estimator will be defined by

$$
C_{p m}=\frac{U S L-L S L}{6 \sqrt{(\bar{D})^{2}+(\overline{\bar{x}}-T)^{2}}}
$$

The $C_{p m k}$ index based on Downton estimator will be defined by

$$
C_{p m k}=\frac{\min (U S L-\overline{\bar{x}}, \overline{\bar{x}}-L S L)}{3 \sqrt{(\bar{D})^{2}+(\overline{\bar{x}}-T)^{2}}}
$$

Where $T=\frac{U S L+L S L}{2}$

\section{APPLICATION}

We collected the data from the factory (Coca-Cola /Erbil) and the data representing the quality properties of drink $(750 \mathrm{ml})$ for Coca-Cola product. We used (100) observations drink $(750 \mathrm{ml})$ for Coca-Cola product, and divided 




\section{العدد (39) حزيران - يونيو 2019}

into (25) samples and each sample consisting of (4) observations as shown in table (1):

Table1: drink $(750 \mathrm{ml})$ for (Coca-Cola /Erbil) product

\begin{tabular}{|c|c|c|c|c|c|c|c|}
\hline sub groups & $\mathrm{x} 1$ & $\mathrm{x} 2$ & $\mathrm{x} 3$ & $\mathrm{x} 4$ & $\mathrm{x}$-bar & S & $\mathrm{D}$ \\
\hline 1 & 750.14 & 750.36 & 750.36 & 751.36 & 750.555 & 0.546596 & 0.540598 \\
\hline 2 & 750.78 & 750.86 & 751.86 & 751.96 & 751.365 & 0.63148 & 0.669987 \\
\hline 3 & 751.12 & 751.22 & 751.28 & 751.38 & 751.25 & 0.108934 & 0.124036 \\
\hline 4 & 750.02 & 750.36 & 750.88 & 751.28 & 750.635 & 0.556747 & 0.637822 \\
\hline 5 & 750.48 & 750.48 & 750.5 & 750.56 & 750.505 & 0.037859 & 0.038391 \\
\hline 6 & 750.08 & 750.2 & 750.2 & 751.04 & 750.38 & 0.443621 & 0.425389 \\
\hline 7 & 750.4 & 750.42 & 750.7 & 750.86 & 750.595 & 0.223532 & 0.245024 \\
\hline 8 & 749.56 & 750.02 & 750.2 & 750.2 & 749.995 & 0.302159 & 0.310073 \\
\hline 9 & 750.34 & 750.49 & 750.7 & 751.02 & 750.6375 & 0.294661 & 0.332211 \\
\hline 10 & 750.5 & 750.54 & 750.54 & 750.66 & 750.56 & 0.069282 & 0.070898 \\
\hline 11 & 750.52 & 750.62 & 750.66 & 750.9 & 750.675 & 0.161142 & 0.174268 \\
\hline 12 & 750.16 & 750.42 & 750.74 & 750.9 & 750.555 & 0.330404 & 0.37498 \\
\hline 13 & 750.34 & 750.48 & 750.54 & 750.6 & 750.49 & 0.111355 & 0.124036 \\
\hline 14 & 750.32 & 750.44 & 750.68 & 751.78 & 750.805 & 0.667008 & 0.682253 \\
\hline 15 & 750.6 & 750.62 & 751.28 & 751.7 & 751.05 & 0.536284 & 0.58452 \\
\hline 16 & 750.26 & 750.3 & 750.52 & 750.62 & 750.425 & 0.173109 & 0.191886 \\
\hline 17 & 750.24 & 750.84 & 750.96 & 751.58 & 750.905 & 0.549272 & 0.611425 \\
\hline 18 & 750.16 & 750.52 & 750.92 & 750.98 & 750.645 & 0.382405 & 0.422198 \\
\hline 19 & 750.54 & 750.62 & 750.8 & 751 & 750.74 & 0.204613 & 0.230313 \\
\hline 20 & 750.69 & 751.4 & 752.18 & 752.36 & 751.6575 & 0.76787 & 0.854748 \\
\hline 21 & 750.32 & 750.9 & 751.32 & 752.16 & 751.175 & 0.774145 & 0.877116 \\
\hline 22 & 750.8 & 750.8 & 751.08 & 751.44 & 751.03 & 0.303535 & 0.324784 \\
\hline 23 & 750.12 & 750.28 & 750.22 & 750.56 & 750.295 & 0.188591 & 0.186143 \\
\hline 24 & 750.26 & 750.4 & 750.44 & 750.54 & 750.41 & 0.116046 & 0.129956 \\
\hline 25 & 750.44 & 750.5 & 750.5 & 750.56 & 750.5 & 0.04899 & 0.053174 \\
\hline
\end{tabular}

$$
\bar{D}=\frac{\sum_{i=1}^{m} D_{i}}{m}=0.36
$$$$
\hat{\sigma}=\frac{\bar{s}}{c_{4}}=\frac{0.3411}{0.9213}=0.3702
$$

$\therefore \bar{D}<\hat{\sigma}$ 


\section{Construction Control Charts for data}

Both charts are used for controlling the mean level in the data of (drink (750 $\mathrm{ml})$ ). The horizontal axis of the chart represents samples sequence, while the vertical axis represents the quality characteristic.

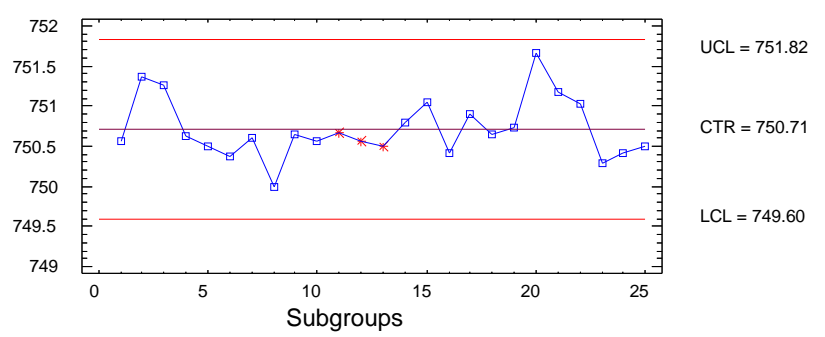

Figure (1): X -bar chart based on $(\hat{\sigma})$

Figure (1) shows that all the points are fallen within the limits of control. This means that the above chart can be relied upon and used in the future for the same properties of quality from which we obtained the data for the purpose of control and monitoring of future production.

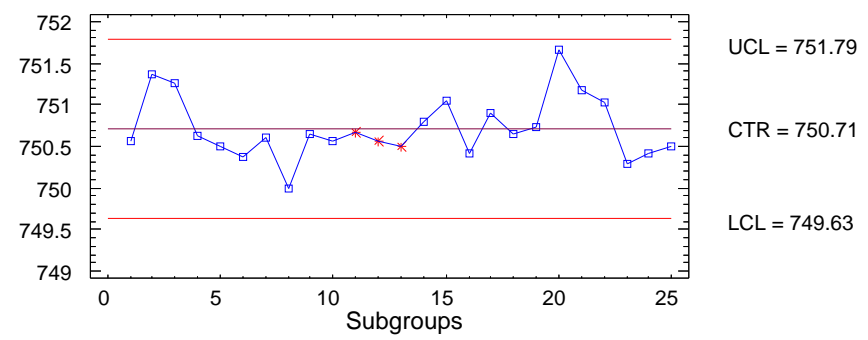

Figure (2): X -Bar chart based on Downton estimator

Figure (2) shows that all the points are fallen within the limits of control. This means that the above chart can be relied upon and used in the future for the same properties of quality from which we obtained the data for the purpose of control and monitoring of future production. 


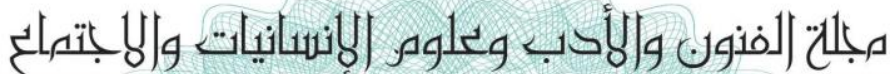

\section{العدد (39) حزيران - يونيو 2019}

Table2.Comparison between Capability Indices Based on Downton estimator and Capability Indices Based on $(\hat{\sigma})$

\begin{tabular}{|c|c|}
\hline Downton estimator & $(\hat{\sigma})$ estimator \\
\hline$C_{p}=\frac{U S L-L S L}{6(\bar{D})}=\frac{752.36-749.56}{6(0.36)}=1.3>1$ & $C_{p}=\frac{U S L-L S L}{6(\hat{\sigma})}=\frac{752.36-749.56}{6(0.3702)}=1.2>1$ \\
\hline $\begin{array}{l}C_{p k}=\min \left(\frac{U S L-\overline{\bar{x}}}{3(\bar{D})}, \frac{\overline{\bar{x}}-L S L}{3(\bar{D})}\right) \\
C_{p k}=\min \left(\frac{752.36-750.71}{3(0.36)}, \frac{750.71-749.56}{3(0.36)}\right) \\
C_{p k}=\min (1.53,1.06) \\
C_{p k}=1.06>1\end{array}$ & $\begin{array}{l}C_{p k}=\min \left(\frac{U S L-\overline{\bar{x}}}{3(\hat{\sigma})}, \frac{\overline{\bar{x}}-L S L}{3(\hat{\sigma})}\right) \\
C_{p k}=\min \left(\frac{752.36-750.71}{3(0.3702)}, \frac{750.71-749.56}{3(0.3702)}\right) \\
C_{p k}=\min (1.48,1.03) \\
C_{p k}=1.03>1\end{array}$ \\
\hline $\begin{array}{l}C_{p m}=\frac{U S L-L S L}{6 \sqrt{(\bar{D})^{2}+(\overline{\bar{x}}-T)^{2}}} \\
C_{p m}=\frac{752.36-749.56}{6 \sqrt{(0.36)^{2}+(750.71-750.96)^{2}}} \\
C=1.07>1\end{array}$ & $\begin{array}{l}C_{p m}=\frac{U S L-L S L}{6 \sqrt{\hat{\sigma}+(\overline{\bar{x}}-T)^{2}}} \\
C_{p m}=\frac{752.36-749.56}{6 \sqrt{(0.3702)^{2}+(750.71-750.96)^{2}}} \\
C_{p m}=1.04>1\end{array}$ \\
\hline$C_{p m k}=\frac{\min (U S L-\overline{\bar{x}}, \overline{\bar{x}}-L S L)}{3 \sqrt{(\bar{D})^{2}+(\overline{\bar{x}}-T)^{2}}}$ & $C_{p m k}=\frac{\min (U S L-\overline{\bar{x}}, \overline{\bar{x}}-L S L)}{3 \sqrt{(\hat{\sigma})^{2}+(\overline{\bar{x}}-T)^{2}}}$ \\
\hline $\begin{array}{l}C_{p m k}=\frac{\min (1.65,1.15)}{3 \sqrt{(0.36)^{2}+(750.71-750.96)^{2}}} \\
C_{p m k}=0.88<1\end{array}$ & $\begin{array}{l}C_{p m k}=\frac{\min (1.53,1.15)}{3 \sqrt{(0.36)^{2}+(750.71-750.96)^{2}}} \\
C_{p m k}=0.85<1\end{array}$ \\
\hline
\end{tabular}




\section{II.CONCLUSION}

1- When we compared scale estimate, Downton estimator have better property than the other $(\hat{\sigma})$ estimator. Reflect that the process Capability Indices values based on Downton estimator greater than the process Capability Indices values based on $(\hat{\sigma})$ estimator that is mean Downton estimator have better property than the other $(\hat{\sigma})$ estimator.It is recommended to use proposed Downton estimator as an alternative to $(\hat{\sigma})$ estimator.

2- The distance between the upper and lower limits in the control chart based on Downton estimator is less than the control chart based on $(\hat{\sigma})$ estimator. This means that the Downton estimator is an important estimate of reducing the size of the error go so far as to.

\section{REFERENCES}

1. Adeoti, O. A, Olaomi, J.O. \& Adekeye, K.S. 2016. Control chart limits for monitoring process mean based on Downton estimator. Quality and Reliability Engineering International, 32(5), pp. $1731-1740$.

2. Abbasi, S.A. \& Miller, A. 2013. D Chart: An Efficient Alternative to Monitor Process Dispersion. Proceedings of the World Congress on Engineering and Computer Science 2011 Vol II WCECS 2011, October 19-21, 2011, San Francisco, USA.

3. Barnett, F.C., Mullen, K. \& Saw, J.G. 1967. Linear estimates of a population scale parameter. Biometrika, 54, pp. 551-554.

4. Chan, L.K., A new measure of process capability: Cpm Cheng, S.W. \& Spiring, F.A. 1988. Journal of Quality Technology, 20(3), pp. 162-175.

5. Downton, F. 1966. Linear estimates with polynomial coefficients. Biometrika, 53, pp. 129-141.

6. Kane, V. E. (1988). Process Capability Indices. J. of Quality Technology 18, 4152. 


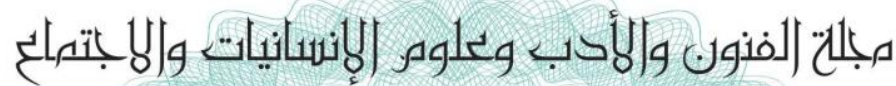

العدد (39) حزيران - يونيو 2019

7. Kotz, S. \& Johnson, N. L. (2002): "Processes Capability Indices-A Review, 19922000". Journal of Quality Technology. Vol. (34), No. (1), pp. 40-42. 